\title{
An Analysis of IEEE 802.11 DCF and Its Application to Energy-Efficient Relaying in Multihop Wireless Networks
}

\author{
Canan Aydogdu, Student Member, IEEE, and Ezhan Karasan, Member, IEEE
}

\begin{abstract}
We present an analytical model for the IEEE 802.11 DCF in multihop wireless networks that considers hidden terminals and accurately works for a large range of traffic loads. An energy model, which considers energy consumption due to collisions, retransmissions, exponential backoff and freezing mechanisms, and overhearing of nodes, and the proposed IEEE 802.11 DCF analytical model are used to analyze the energy consumption of various relaying strategies. The results show that the energy-efficient relaying strategy depends significantly on the traffic load. Under light traffic, energy spent during idle mode dominates, making any relaying strategy nearly optimal. Under moderate traffic, energy spent during idle and receive modes dominates and multihop transmissions become more advantageous where the optimal hop number varies with processing power consumed at relay nodes. Under very heavy traffic, where multihopping becomes unstable due to increased collisions, direct transmission becomes more energy efficient. The choice of relaying strategy is observed to affect energy efficiency more for large and homogeneous networks where it is beneficial to use multiple short hops each covering similar distances. The results indicate that a cross-layered relaying approach, which dynamically changes the relaying strategy, can substantially save energy as the network traffic load changes in time.
\end{abstract}

Index Terms-IEEE 802.11 DCF, analytical model, energy-efficient relaying, multihop wireless networks, hidden terminal.

\section{INTRODUCTION}

$\mathrm{O}$ NE of the greatest limitations of wireless multihop networks is the finite energy resource of terminals. As a result, energy-efficient routing protocol design for wireless multihop networks has been the focus of many research efforts. Many minimum-energy routing protocols consider solely the energy consumption at the amplifier due to transmission, ignoring the energy consumed at the transmitter and receiver circuitry for reception and processing, favoring multihop paths [1], [2]. However signal processing associated with packet transmission and reception consumes nonnegligible energy [3]. It has been shown that routing through multiple short hops is not always more energy efficient than direct transmission when processing power is also taken into account [4], [5], [6], [7].

Recent studies have shown that energy efficiency of routing protocols is enhanced by cross layering, which considers multiple protocol layers and allows exchange of information among layers [8], [9], [10]. This paper extends the studies investigating the effect of relaying on energy efficiency using a cross-layered approach. The effects of medium access control (MAC) contention on energy efficiency are investigated to answer the following basic question: "When should we use a single long hop or multiple short hops for energy-efficient routing in wireless multihop networks?" The goal of this study is to reveal when multihopping

- The authors are with the Department of Electrical and Electronics Engineering, Bilkent University, Bilkent 06800, Ankara, Turkey.

E-mail: \{canan,ezhan\}@ee.bilkent.edu.tr.

Manuscript received 1 Sept. 2009; revised 4 Oct. 2010; accepted 7 Oct. 2010; published online 17 Dec. 2010.

For information on obtaining reprints of this article, please send e-mail to: tmc@computer.org, and reference IEEECS Log Number TMC-2009-09-0359. Digital Object Identifier no. 10.1109/TMC.2010.239. becomes advantageous and to state guidelines for energyefficient routing considering MAC contention.

Under transmit power control, the energy consumed at the physical (PHY) layer decreases when switching from direct transmission to multihopping, which in turn decreases the number of contending stations within the transmission range. A decreased number of contending stations implies decreased number of collisions, retransmissions, backoff, and freezing mechanisms at the MAC layer, and less overhearing, which decrease the overall energy consumption. On the other hand, multihopping requires successful transmissions at all hops of the path and energy is lost when packet is lost at some hop. The relaying strategy used by the routing protocol at the network layer has impacts on MAC parameters, affecting energy efficiency. Thus, for energy-efficient selection of routes, the behavior of MAC layer should be carefully contemplated.

The IEEE 802.11 distributed coordination function (DCF) is studied in this paper, since most commercial wireless products are based on the IEEE 802.11 standard [11]. The IEEE 802.11 DCF performance changes when switching from local area networks to wireless multihop networks in three major aspects: 1) paths are multihop whereas they are single hop in local area networks, 2) traffic handled by each node is the superposition of node's own traffic and the relay traffic, and 3) the behavior of each node depends also on hidden nodes.

Various analytical models exist for IEEE 802.11 DCF based single-hop networks, where the first model is introduced for saturated networks with infinite retry count [12]. Further models have been developed under different assumptions such as finite retry limit [13], unsaturated traffic conditions [14], etc. On the other hand, there are few 
analytical models for multihop networks, which work under either unsaturated [15] or saturated [16], [17], [18] traffic conditions. Owing to the comparable complexity increase when switching from single hop to multihop network architecture, the existing models are based on simplified assumptions. The hidden terminal problem is eliminated in [15] by the assumption of RTS/CTS mode with busy tone, whereas the analysis in [16] ignores the existence of hidden terminals and assumes that each node is either relay or source. The hidden terminal problem is included in the analysis of IEEE 802.11 DCF in [17] and [18], where only 3-node and string topologies are considered, respectively. An analytical DCF model, which considers hidden terminals and works for various traffic loads in various multihop topologies, does not exist to the best of our knowledge.

Energy consumption of IEEE 802.11 DCF in single-hop networks is analyzed in [19], [20], [21], [22], [23]. The binary exponential backoff mechanism (BEB) is ignored in [19], whereas BEB is approximated by a $p$-persistent model in [20] for saturated traffic conditions. Analytical energy models which consider the BEB and account for the IEEE 802.11 DCF protocol by encapsulating the carrier sensing, collisions, and freezing mechanism in backoff are proposed with infinite retry limit in [21] and finite retry limit in [22] and [23] under saturated traffic conditions. Despite various models for energy consumption in single-hop networks [19], [20], [21], [22], [23], energy consumption in IEEE 802.11 DCF based wireless multi-hop networks has not been analytically modeled so far.

The primary contribution of this study is an analytical framework for the investigation of the energy efficiency of relaying strategies in IEEE 802.11 DCF based wireless multihop networks. To the best of our knowledge, this is the first study in multihop networks that includes the energy consumption due to MAC operations such as collisions, retransmissions, overhearing of nodes, $\mathrm{BEB}$, and freezing mechanisms. Owing to the fact that existing models are inadequate for energy-efficiency analysis in multihop networks, an analytical model for IEEE 802.11 DCF for multihop networks is introduced which:

- considers hidden terminals,

- provides fairly accurate results for large range of traffic loads,

- works for any given two-dimensional topology,

- increases the accuracy and scalability of the analytical model by joint use of fixed and variable slots, and

- allows each node to be both source and/or relay.

A semi-Markov chain (SMC) based model is proposed, since SMC models are shown to predict the DCF behavior better than discrete-time Markov chain (DTMC) models [12], [13]. The analytic results obtained via the IEEE 802.11 DCF and the energy model, supported by simulations, show that the energy-efficient relaying strategy highly depends on the traffic load. Under light traffic, energy spent during idle mode is responsible for most of the energy consumed, making any relaying strategy equivalently optimal. Under moderate traffic, energy spent during idle and receive modes dominates and multihop transmissions become more advantageous. At heavy traffic, multihopping becomes unstable due to increased packet collisions and excessive traffic congestion, and direct transmission becomes more energy efficient and stable. It is also shown that the processing power at relay nodes affects the optimal hop number, but only for a specific range of traffic loads. The major attributes of the proposed analytical IEEE 802.11 DCF model for multihop networks are discussed in Section 2 and the model is introduced in Section 3. A theoretical framework to evaluate the energy consumption of IEEE 802.11 DCF is introduced in Section 4. Finally, analytical and simulation results are presented in Section 5.

\section{Major Attributes of the Proposed ANALYTICAL MODEL FOR IEEE 802.11 DCF}

Owing to the fact that the existing analytical models of IEEE 802.11 DCF systems are inadequate for an energy-efficiency analysis in wireless multihop networks, an analytical model for IEEE 802.11 DCF for wireless multihop networks is developed in this paper. The major attributes of the proposed DCF model together with the limitations and assumptions of the previous DCF models are described in this section.

\subsection{Joint Use of Fixed and Variable Slots}

Most of the IEEE 802.11 DCF models are based on discretizing time into variable length slots, where a slot is either the constant SlotTime, or the variable time interval between two consecutive backoff counter decrements [11]. A limitation stemming from variable slot length is mentioned in [17] and it is shown that the error in finding throughput for various PHY layer parameters grows for smaller contention window sizes, larger packet sizes, and lower data rates. On the other hand, using short fixed length slots in the analysis increases the number of states of the DTMC. In order to limit the number of states, the fixed slot length analysis in [17] is based on constant contention window (CCW) assumption, which hinders the dynamics of the IEEE 802.11 DCF.

In this paper, a solution methodology different from the literature is developed where a joint usage of fixed and variable length slots is adapted. The SMC is developed and solved based on variable slot notion, whereas MAC parameters such as collision probability and network allocation vector (NAV) setting probabilities are developed based on fixed length slot notion. Variable length slot notion ensures that the number of states of the SMC is kept relatively small, so that $B E B$ is included in the analysis, reflecting the real dynamics of the IEEE 802.11 DCF. On the other hand, the fixed-slot notion used in developing MAC parameters ensures that our analysis is not prone to the errors pointed out by Tsertou and Laurenson [17]. This way, the weaknesses of variable slot length analysis are eliminated, while keeping the SMC state size small enough to be computationally efficient even with BEB.

\subsection{From Channel State Probability toward NAV Setting Probability}

In studies modeling the IEEE 802.11 DCF in single-hop networks, successful, collision, and idle channel perceived by a node are represented by channel state probabilities. However this representation is not appropriate for multihop networks, because the channel state perceived by a node may not be the actual state of the channel when hidden nodes 
exist. For example, two concurrent successful transmissions in the channel of a node are perceived as a collision. Also, a node perceives a successful channel if it successfully receives an RTS or CTS frame that collides at the relevant receivers. In [17], instead of distinguishing among channel states the probability of freezing/not freezing the backoff counter is used. But NAV duration takes only one value and leads to insufficient accuracy in large multihop networks. We switch from channel state probability notion to NAV setting probability notion and discriminate between events that set the NAV for long and short durations.

\subsection{Large Range of Traffic Loads}

The existing analytical models for IEEE 802.11 DCF for both single-hop and multihop networks work under either unsaturated [14], [15] or saturated [12], [13], [16], [17], [18] traffic loads. In this paper, we develop an analytical model for multihop networks that operates in any traffic load ranging from light to heavy traffic conditions.

\subsection{Any Given Topology and Traffic Pattern}

Unlike previous studies that have focused on analyzing IEEE 802.11 behavior by considering hidden terminals in multihop networks under assumptions of specific network (e.g., [17], [18]), the proposed model accurately works for various network topologies and traffic patterns considered in this study.

\subsection{Not Only RTS Collisions}

Existence of hidden terminals in multihop networks results in collisions other than collision of RTS packets. An RTS or CTS packet that is not received correctly by neighbors inside the carrier sensing range may cause collision of other types of frames. In the analysis carried out in this paper, collisions of RTS, CTS, DATA, or ACK frames with each other are also considered in the calculation of the probability of collision.

\section{IEEE 802.11 DCF MODEL FOR MULTIHOP AD HOC NETWORKS}

Basics of the proposed IEEE 802.11 DCF model is introduced in this section after an overview of assumptions. Geometry related notations, calculation of the state residence times, NAV setting probabilities and collision probability are described next. A list of notations used in this paper is given in Table 1.

\subsection{Assumptions}

Several simplifying assumptions also made by several previous studies [12], [13], [14], [15], [16], [17], [18] are adapted in order to provide an analytically tractable solution to the problem:

1. disc radio model,

2. Poisson offered traffic,

3. bit error-free channel, and

4. stationary nodes.

No assumption is imposed neither on the topology nor on the traffic pattern. In order to be able to compare the energy efficiency of relaying strategies, a comparison is conducted
TABLE 1

List of Notations

\begin{tabular}{|c|c|}
\hline$W_{0}$ & Minimum contention window size \\
\hline$W_{b}$ & The maximum backoff counter value at stage $b$ \\
\hline$b$ & The backoff stage \\
\hline$B$ & The maximum counter value \\
\hline$E P B$ & Energy per bit \\
\hline Everhear & Overhear energy per bit \\
\hline$E_{r x}$ & Receive energy per bit \\
\hline$E_{t x}$ & Transmit energy per bit \\
\hline$h$ & Number of hops of all paths in the network \\
\hline$k$ & The backoff counter value \\
\hline$M$ & Maximum retry count \\
\hline$n$ & Avg. \# of nodes inside the carrier sensing range \\
\hline$N$ & Total number of nodes in the network \\
\hline $\bar{n}_{M}$ & Avg. \# of dropped packets due to exceeding $M$ \\
\hline$N_{d r o p}$ & $\begin{array}{l}\text { Total \# of unsuccessful transmissions per path per suc- } \\
\text { cessful reception }\end{array}$ \\
\hline$N_{\text {rxexc }}$ & Avg. \# of nodes inside $S_{r x e x c}$ \\
\hline$N_{\text {rxint }}$ & Avg. \# of nodes inside $S_{r x i n t}$, including $t x$ and $r x$ \\
\hline$N_{\text {succ }}$ & $\begin{array}{l}\text { Total \# of successful transmissions per path per success- } \\
\text { ful reception }\end{array}$ \\
\hline$p$ & Prob. of collision given transmission occurs \\
\hline$P_{\text {coll }}$ & $\begin{array}{l}\text { Prob. that NAV is set for short duration given that } \\
\text { carrier sensing is done with zero NAV }\end{array}$ \\
\hline$P_{\text {idle }}$ & $\begin{array}{l}\text { Prob. that NAV is not set given that carrier sensing is } \\
\text { done with zero NAV }\end{array}$ \\
\hline$P_{i f q}(i)$ & Prob. of packet drop at interface queue of node $i$ \\
\hline$P_{\text {succ }}$ & $\begin{array}{l}\text { Prob. that NAV is set for long duration given that carrier } \\
\text { sensing is done with zero NAV }\end{array}$ \\
\hline$P w r_{i d l e}$ & Idle power in Watts \\
\hline$P w r_{r x}$ & Receive power in Watts \\
\hline$P w r_{t x}$ & Transmit power in Watts \\
\hline$q$ & Prob. that IFQ is empty upon processing a packet \\
\hline$R_{e x c}$ & The ratio of \# of nodes inside $S_{e x c}^{t x \rightarrow i}$ to $n$ \\
\hline$R_{\text {excSrxex }}$ & The ratio of \# of nodes inside $S_{\text {excSrxexc }}$ to $n$ \\
\hline$R_{\text {intSrxexc }}$ & The ratio of \# of nodes inside $S_{\text {intSrxexc }}$ to $n$ \\
\hline$R_{\text {intSrxint }}$ & The ratio of \# of nodes inside $S_{\text {intsrxint }}$ to $n$ \\
\hline$R_{r x \text { Srxexc }}$ & The ratio of \# of nodes inside $S_{r x S r x e x c}$ to $n$ \\
\hline$R_{t x S r x e x c}$ & The ratio of \# of nodes inside $S_{t x S r x e x c}$ to $n$ \\
\hline$R_{t x \text { Srxint }}$ & The ratio of \# of nodes inside $S_{t x \text { Srxint }}$ to $n$ \\
\hline$S_{e x c}^{t x \rightarrow i}$ & Carrier sensing area of $i \in S_{t x}$, not exposed to $t x$ \\
\hline$S_{i n t}^{t x \rightarrow i}$ & Intersection of carrier sensing areas of $t x$ and $i \in S_{t x}$ \\
\hline$S_{\text {rxint }}$ & Intersection of carrier sensing regions of $t x$ and $r x$ \\
\hline$S_{\text {rxexc }}$ & Receiver exclusive region \\
\hline$S_{t x}$ & The carrier sensing region of the transmitter $t x$ \\
\hline$T_{b u s y}$ & Total busy duration per path per successful reception \\
\hline$T_{\text {drop }}$ & Busy duration of one unsuccessful transmission \\
\hline$T_{e}$ & Avg. time spent during backoff with empty queue \\
\hline$T_{r c}$ & State residence time of receive collision states \\
\hline$T_{r s}$ & State residence time of receive success states \\
\hline$T_{\text {succ }}$ & The busy duration of one successful transmission \\
\hline$T_{t c}$ & State residence time of transmit collision states \\
\hline$T_{t s}$ & State residence time of transmit success states \\
\hline$\pi_{i d l e}$ & Sum of steady state probabilities of idle states \\
\hline$\pi_{r c}$ & Sum of steady state prob. of receive collision states \\
\hline$\pi_{r s}$ & Sum of steady state prob. of receive success states \\
\hline$\pi_{t c}$ & Sum of steady state prob. of transmit collision states \\
\hline$\pi_{t s}$ & Sum of steady state prob. of transmit success states \\
\hline$\lambda_{t}(i)$ & Average packet arrival rate at node $i$ \\
\hline$\lambda_{o}(i)$ & Average packet generation rate at node $i$ \\
\hline$\lambda_{r}(i)$ & Average total relay traffic at node $i$ \\
\hline$\tau$ & Prob. of transmission \\
\hline$\sigma$ & The state residence time of idle states (SlotTime) \\
\hline $\bar{\sigma}$ & Average NAV duration \\
\hline
\end{tabular}

using the same topology and traffic pattern where all nodes in the network are assumed to adapt the same routing strategy: each generated packet traverses a path of $h$ hops. Thus, source-destination pairs are selected so that a reasonable $h$-hop path exists. Nodes are assumed to conduct power control with infinitely variable levels and 


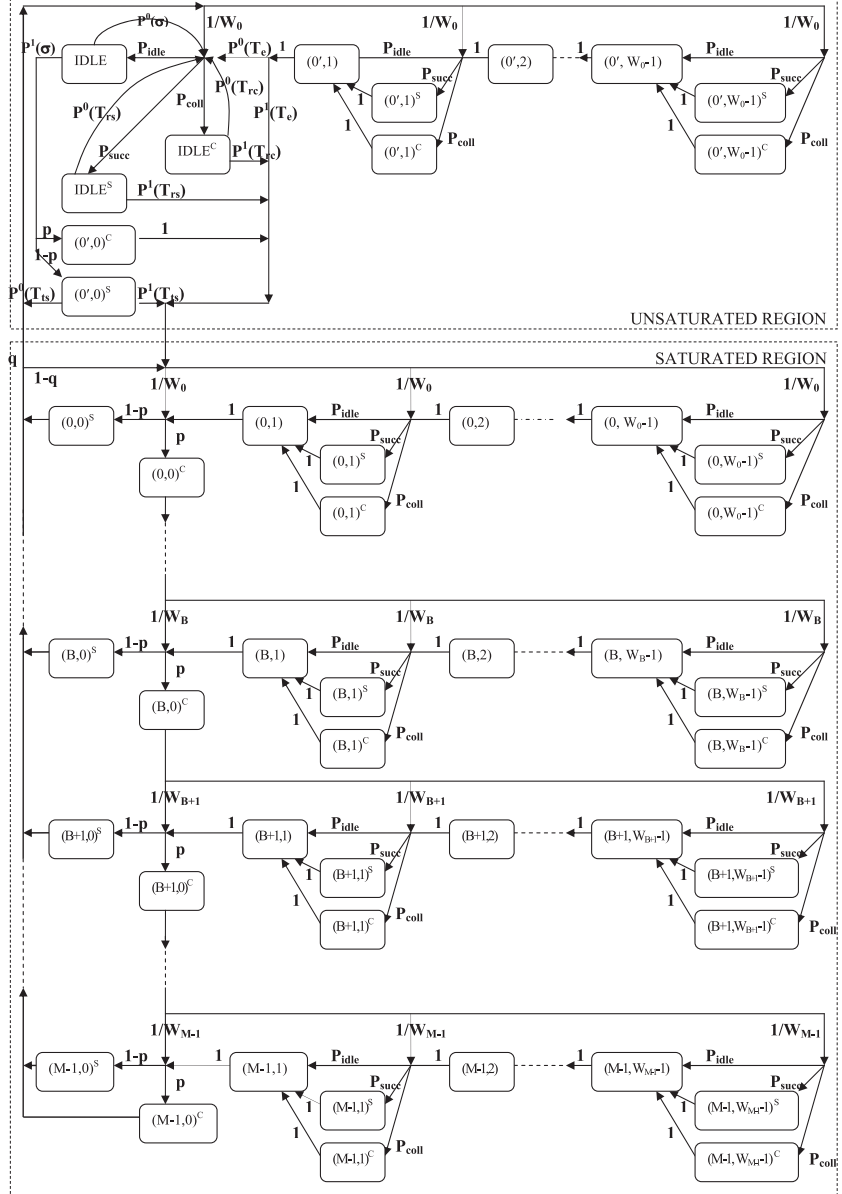

Fig. 1. SMC model for the IEEE 802.11 DCF based node.

transmit with the minimum required power to reach the next hop. The transmission range is assumed to be equal to the carrier sensing range and the interference range.

Each node is assumed to use the IEEE 802.11 DCF in conjunction with the RTS/CTS exchange as the MAC protocol. Although the RTS/CTS handshake mechanism introduces an overhead, it is shown to improve IEEE 802.11 performance in multihop wireless networks when hidden nodes are present [24]. In case of a collision, packets are retransmitted according to BEB until the maximum retry count $(M)$ is reached. At each transmission attempt, regardless of the number of retransmissions, each packet collides with a conditional probability $p$, conditioned on the fact that the particular node is attempting a transmission. Packets are dropped after $M$ unsuccessful retries with probability $p^{M}$ or due to overflow of the finite sized interface queue (IFQ) with probability $P_{i f q}(i)$ at node $i$ for $1 \leq i \leq N$, where $N$ is the total number of nodes in the wireless network. In our model, a single value is used for $p$ which corresponds to the average conditional collision probability taken over all links, whereas $P_{i f q}(i)$ is different for each node.

\subsection{Basics of IEEE 802.11 DCF Model}

The IEEE 802.11 DCF behavior of a node is modeled by the SMC given in Fig. 1, which captures both the transmit and the receive states of the node. The state diagram is Markov since the future state of the node given the present state is independent of the past state; and it is semi-Markov due to state dependent residence times [25]. The SMC is twodimensional, where the first dimension is the backoff stage and the second is the backoff counter value. Backoff states of the SMC are represented by the notation $(b, k)$, where $b$ is the backoff stage and $k$ is the backoff counter value. The maximum backoff stage is limited by $M$ and the maximum counter value is limited by $B$. The maximum backoff counter value at stage $b$ is $W_{b}$ where,

$$
W_{b}= \begin{cases}W_{0}, & b=0^{\prime}, \\ 2^{b} W_{0}, & 0 \leq b<B, \\ 2^{B} W_{0}, & B \leq b<M .\end{cases}
$$

$b=0^{\prime}$ corresponds to the backoff stage when the IFQ is empty. The backoff states at which the backoff counter is frozen are represented by $(b, k)^{S}$ and $(b, k)^{C}$, for $k \neq 0$, where NAV is set for a long and short duration, respectively. The states of the SMC are grouped in five categories according to state residence times as follows:

1. Idle states. The $I D L E$ state where the node is idle waiting in unsaturated region and the backoff states $(b, k)$, for $b \in\left\{0^{\prime},[0, M)\right\}$ and $k \in\left[1, W_{b}\right)$, are called as idle states. The state residence time of idle states is denoted by $\sigma$, which is also equal to a SlotTime.

2. Transmit success states. These are the states $(b, 0)^{S}$ for $b \in\left\{0^{\prime},[0, M)\right\}$ with state residence time $T_{t s}$ where a successful transmission occurs.

3. Transmit collision states. These are the states $(b, 0)^{C}$ for $b \in\left\{0^{\prime},[0, M)\right\}$ with state residence time $T_{t c}$, where a collision occurs.

4. Receive success states. These are the states $I D L E^{S}$ and $(b, k)^{S}$ for $b \in\left\{0^{\prime},[0, M)\right\}$ and $k \in\left[1, W_{b}\right)$ with state residence time $T_{r s}$, where the NAV is set and the backoff counter is frozen for at least one DATA reception duration.

5. Receive collision states. These are the states $I D L E^{C}$ and $(b, k)^{C}$ for $b \in\left\{0^{\prime},[0, M)\right\}$ and $k \in\left[1, W_{b}\right)$ with residence time $T_{r c}$, where NAV is set and contains no DATA reception.

The steady state probabilities of being in idle, transmit success, transmit collision, receive success, and receive collision state categories are represented by $\pi_{i d l e}, \pi_{t s}, \pi_{t c}, \pi_{r s}$, and $\pi_{r c}$, respectively, and are calculated by summing up the steady state probabilities of all the states in the corresponding category. The reader is referred to [26] for a detailed description of states and state transition probabilities of the SMC given in Fig. 1.

$T_{e}$ is the average time spent during the backoff stages $\left(0^{\prime}, k\right)$ for $k \in\left[1, W_{0}\right)$ and is calculated by $T_{e}=\frac{W_{0} \bar{\sigma}}{2}$, where $\bar{\sigma}$ is the average NAV duration given by

$$
\bar{\sigma}=P_{\text {succ }}\left(T_{r s}+\sigma\right)+P_{\text {coll }}\left(T_{r c}+\sigma\right)+P_{\text {idle }} \sigma .
$$

The channel state probability notion introduced in analytical modeling of DCF for single-hop networks is transformed here into NAV setting probability notion for multihop networks due to existence of hidden terminals. The decision on setting NAV is given at certain instants of time as shown in Fig. 2, corresponding to time instants when the node does carrier sensing and the NAV is zero. Thus NAV setting probabilities are conditioned on the fact that the node does carrier sensing with zero NAV. Three NAV setting 


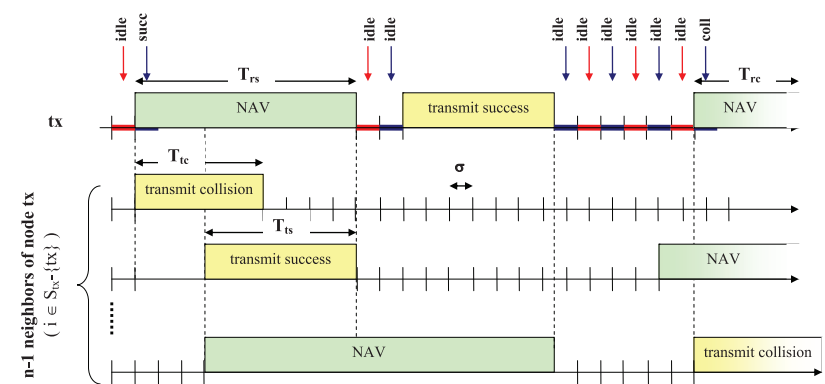

Fig. 2. Illustration of time instants at which NAV setting probabilities $P_{\text {idle }}, P_{\text {succ }}$, and $P_{\text {coll }}$ are calculated.

probabilities are defined: 1) $P_{\text {idle }}$ is the probability that NAV is not set, 2) $P_{\text {succ }}$ is the probability that NAV is set for a long duration that contains at least one DATA reception, and 3) $P_{\text {coll }}$ is the probability that NAV is set for a short duration that does not contain any DATA reception.

The probability of transmission, $\tau$, introduced in [12], is calculated by the summation of steady state probabilities of the transmit success and the transmit collision states of DTMC given in Fig. 1. Note that, the summation of the steady state probabilities of the transmit states of the DTMC gives the probability of transmission whereas the same summation for the SMC gives the probability that a node is transmitting.

The conditional collision probability $p$ is different for each link, since the links in the network are not homogeneous. This leads to a computationally untractable analytical model, where each link should be modeled by a separate SMC and SMC's as many as links is to be solved in parallel at each round of fixed point iterations. In order to simplify the analysis, an average $p$ is found which is averaged over all links weighted by the traffic carried over each link. Extensive simulations conducted with various topologies and traffic patterns, excluded in this paper due to space limitation, demonstrate that finding an average $p$ in the analysis results with reasonable errors while maintaining tractable computations for regular topologies, whereas the error increases with the irregularity of the topology and traffic pattern.

The packet arrivals to IFQ at a node follow the Poisson process with average rate $\lambda_{t}$. Packets are served using the first in first out discipline by a single server. The service time is a nonnegative random variable denoted by random variable $T_{S}$, which has a discrete probability of $\operatorname{Pr}\left(T_{S}=\right.$ $\left.t_{s}(i)\right)$ for $T_{S}$ being $t_{s}(i)$, expressed as

$$
\begin{aligned}
& \operatorname{Pr}\left\{T_{S}=t_{s}(i)\right\}= \begin{cases}(1-p) p^{i}, & \text { if } 0 \leq i<M, \\
p^{M}, & \text { if } i=M,\end{cases} \\
& t_{s}(i)= \begin{cases}T_{t s}+i T_{t c}+\sum_{j}^{i+1} W_{j} \frac{\bar{\sigma}}{2}, & \text { if } 0 \leq i<M, \\
M T_{t c}+\sum_{j}^{M} W_{j} \frac{\bar{\sigma}}{2}, & \text { if } i=M .\end{cases}
\end{aligned}
$$

Thus, IFQ can be modeled as an M/G/1/K queue where $\mathrm{K}$ represents the maximum number of packets in the queue, and can be solved by the techniques in [27]. $P_{i f q}$ corresponds to the steady-state probability of $K$ packets in the queuing system, whereas $q$ corresponds to the probability that the node's buffer is empty after the node finishes processing a packet in backoff [26].

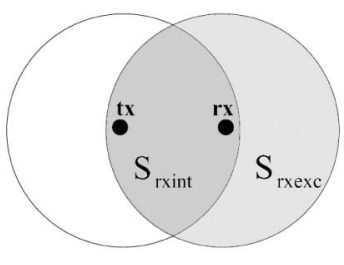

(a)

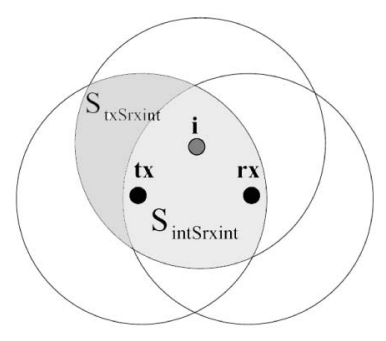

(c)

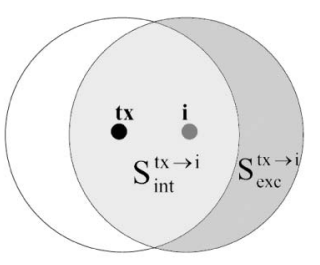

(b)

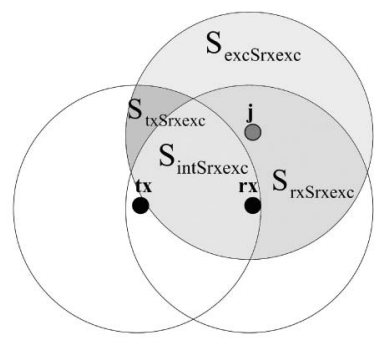

(d)
Fig. 3. Illustration of carrier sensing regions (a) $S_{r x e x c}$ and $S_{r x i n t}$, (b) $S_{e x c}^{t x \rightarrow i}$ and $S_{i n t}^{t x \rightarrow i}$, (c) $S_{\text {txSrxint }}$ and $S_{\text {intSrxint }}$ formed by nodes $t x, r x$ and $i \in S_{\text {rxint }}-\{t x\}$, and (d) $S_{\text {txSrxexc }}, S_{\text {rxSrxexc }}, S_{\text {intSrxexc }}$, and $S_{\text {excSrxexc }}$ formed by nodes $t x, r x$, and $j \in S_{\text {rxexc }}$.

Between each node pair $(i, j)$ in the network there is a Poisson traffic with rate $\lambda_{o}(i, j)$. The total traffic at node $i$ is given by $\lambda_{t}(i)=\lambda_{o}(i)+\lambda_{r}(i)$, where $\lambda_{o}(i)=\sum_{j} \lambda_{o}(i, j)$ and $\lambda_{r}(i)$ is the total relay traffic. Let $P_{k l}$ be the sequence of nodes traversed by the path between nodes $k$ and $l$. For $i \in P_{k l}$, define the set $Q_{k l i}$ as the set of nodes on path $P_{k l}$ that precede node $i . \lambda_{r}(i)$ is calculated by summing up all the relay traffic crossing node $i$ :

$$
\lambda_{r}(i)=\sum_{k, l \neq i: i \in P_{k l}} \lambda_{o}(k, l)\left(1-p^{M}\right)^{\left|Q_{k l i}\right|} \prod_{j \in Q_{k l i}}\left(1-P_{i f q}(j)\right) .
$$

The relay traffic is not Poisson because of packet drops at the IFQ and at the intermediate nodes due to the retry limit. In order to simplify the analysis, we assume that the relay traffic is Poisson and hence the overall traffic arriving at a node is Poisson with rate $\lambda_{t}(i)$. The probability of receiving no packets and receiving one or more packets in a duration of $t$ is denoted by $P^{0}(t)$ and $P^{1}(t)$, respectively.

The analytic model is solved through fixed point iterations, since no closed form solution exists. The convergence of the fixed point iterations is not investigated and left as a future work, but for all the scenarios that we studied, the fixed point iterations always converged.

\subsection{Geometry Related Notations}

The carrier sensing region of a transmitter $t x$ is denoted by $S_{t x}$. A fixed number of $n$ nodes are assumed to be within this carrier sensing region of each node. For a transmission from $t x$ to a receiver $r x$, the part of a receiver carrier sensing region which is not exposed to the transmitter carrier is called the receiver exclusive region [15], and is denoted by $S_{\text {rxexc }}$ (Fig. 3a). The average number of nodes inside $S_{\text {rxexc }}$ is denoted by $N_{\text {rxexc }}$. The intersection area of carrier sensing regions of $t x$ and $r x$ is denoted by $S_{\text {rxint }}$. The average number of nodes inside $S_{\text {rxint }}$, including $t x$ and $r x$, is denoted by $N_{\text {rxint }}$. Although the variables $n, N_{\text {rxexc, }}$ and $N_{r x i n t}$ are different for each $t x-r x$ pair for an arbitrary 
topology, average values obtained via a traffic weighted averaging operation over all links in the network are used.

The part of the carrier sensing region of any node $i \in S_{t x}$, which is not exposed to the transmitter carrier is denoted by $S_{e x c}^{t x \rightarrow i}$ (Fig. 3b), whereas the intersection area of carrier sensing regions of $t x$ and node $i \in S_{t x}$ is denoted by $S_{i n t}^{t x \rightarrow i}$. The number of possible intended receivers of a node $j$ that are inside the region $S_{e x c}^{i \rightarrow j}$ is denoted by $n t x_{e x c}^{i \rightarrow j}$. $S_{t x \text { Srxint }}$ and $S_{\text {intSrxint }}$ are the regions formed by borders of carrier sensing regions of nodes $t x, r x$, and $i \in S_{\text {rxint }}-\{t x\}$ (Fig. 3c), whereas $S_{\text {txSrxexc, }} S_{\text {rxSrxexc, }} S_{\text {intSrxexc, }}$ and $S_{\text {excSrxexc }}$ are the regions determined by the borders of carrier sensing regions of nodes $t x, r x$, and $j \in S_{\text {rxexc }}$ (Fig. 3d). The ratio of number of nodes inside regions $S_{\text {txSrxint }}, S_{\text {intSrxint }}, S_{\text {txSrxexc }}, S_{\text {rxSrxexc }}, S_{\text {intSrxexc, }}$ and $S_{\text {excSrxexc }}$ to $n$ are represented by $R_{\text {txSrxint }}, R_{\text {intSrxint }}$, $R_{\text {txSrxexc }}, R_{\text {rxSrxexc }}, R_{\text {intSrxexc }}$, and $R_{\text {excSrxexc }}$, respectively.

\subsection{State Residence Times}

The residence times of a node in transmit states are

$$
\begin{aligned}
& T_{t s}=T_{R T S}+T_{C T S}+T_{D A T A}+T_{A C K}+3 S I F S+D I F S, \\
& T_{t c}=T_{R T S}+C T \text { Stimeout }+ \text { DIFS, }
\end{aligned}
$$

where $T_{R T S}, T_{C T S}, T_{D A T A}$, and $T_{A C K}$ correspond to transmission times of RTS, CTS, DATA, and ACK packets, respectively, and CTStimeout is the CTS timeout duration [11]. $T_{t s}$ cannot be extended by transmissions of neighbor nodes in range, since no node is allowed to send anything until the end of the reception of ACK plus a carrier sensing duration of DIFS. Likewise, $T_{t c}$ cannot be extended by neighbor transmissions, since the the source node of collision is not allowed to receive anything during CTSTimeout. Note that DATA collisions are neglected in the calculation of $T_{t c}$.

In case the node receives a successful or collided DATA destined for itself or the NAV is set by one DATA reception, the receive success state residence time is equal to $T_{t s}$. We neglect the time difference of $T_{R T S}+S I F S$ in case the node is the receiver of a successful transmission or the NAV is set by a CTS frame. Assuming that at most two DATA receptions may overlap under heavy traffic with probability $1-q, T_{r s}$ is calculated as $T_{r s}=T_{t s}+(1-q) T_{t s} / 2$.

In case the node is the receiver of a collision, NAV is set by $T_{r c}$. Assuming at most two collision overlaps, $T_{r c}$ is calculated as $T_{r c}=1.5 R T S+E I F S+(1-q) E I F S / 2$. The collision of DATA packet when the node is the intended receiver is neglected in the calculation of $T_{r c}$, so that a collision is extended by half RTS duration on the average. After each collision, the station waits for EIFS before taking an action and NAV is extended by an average amount of $\frac{E I F S}{2}$ with probability $(1-q)$. Note that, collision of control frames is included in calculation of $T_{r c}$, whereas collision with a DATA frame is included in calculation of $T_{r s}$.

\subsection{Calculation of NAV Setting Probabilities}

Node $t x$ does not set its NAV during a fixed time slot of length $\sigma$, if the $n-1$ nodes inside $S_{t x}$ do not start a transmission. Since the analysis is transmission based rather than packet based, by start of a transmission, we allude the first $\sigma$ portion of an RTS packet of a successful transmission or collision, and the first $\sigma$ portion of a CTS response of a successful reception.
Denoting the probability of starting a successful transmission (collision), i.e., start sending the RTS of a successful transmission or CTS response to a node $i \in S_{e x c}^{t x \rightarrow i}$ (i.e., start sending RTS of a collision), as $\tau_{s}\left(\tau_{c}\right)$, and assuming that the probability of not starting a transmission, $1-\tau_{s}-\tau_{c}$, is same and independent for each of the $n-1$ nodes, $P_{\text {idle }}$, is in the product form and it is expressed as follows:

$$
P_{\text {idle }}=\left(1-\tau_{s}-\tau_{c}\right)^{n-1} .
$$

$\tau_{s}$ and $\tau_{c}$ are obtained by dividing the steady state probabilities of the states at which a successful transmission and a collision is started, respectively, by $\pi_{\text {allowed, }}^{N A V}$ which is the sum of the steady state probabilities of allowed states at the instants of time where node $t x$ is carrier sensing with zero NAV. The derivation given in Appendix A is based on the fixed length slot notion, so that the states of the SMC are divided into states of residence times of $\sigma$. Hence $\tau_{s}$ and $\tau_{c}$ are given by

$$
\begin{aligned}
\tau_{s}= & \frac{1}{\pi_{\text {allowed }}^{N A V}}\left(\pi_{t s} \frac{\sigma}{T_{t s}}+K_{1} \pi_{r s} \frac{\sigma}{T_{t s}}\right), \\
\tau_{c}= & \frac{1}{\pi_{\text {allowed }}^{N A V}} \pi_{t c} \frac{\sigma}{T_{t c}}, \\
\pi_{\text {allowed }}^{N A V}= & 1-\pi_{t s} \frac{T_{t s}-\sigma}{T_{t s}}-\pi_{t c} \frac{T_{t c}-\sigma}{T_{t c}} \\
& -K_{1} \pi_{r s} \frac{T_{t s}-T_{R T S}-S I F S-\sigma}{T_{t s}} \\
& -\left(1-R_{\text {exc }}\right)\left(\pi_{r s} \frac{T_{r s}-\sigma}{T_{r s}}+\pi_{r c} \frac{T_{r c}-\sigma}{T_{r c}}\right),
\end{aligned}
$$

where $K_{1}$, averaged over each node $i \in S_{t x}-\{t x\}$, is the ratio of successful receptions from any node $j \in S_{e x c}^{t x \rightarrow i}$ to the total receptions.

Node $t x$ sets its NAV for a duration that contains at least one DATA reception in case, one node is transmitting either successful or failure and rest of the $n-1$ nodes are not, or at least two successful transmissions occur, corresponding to probability

$$
\begin{aligned}
P_{\text {succ }}= & (n-1)\left(\tau_{s}+\tau_{c}\right)\left(1-\tau_{s}-\tau_{c}\right)^{n-2}+1-\left(1-\tau_{s}\right)^{n-1} \\
& -(n-1) \tau_{s}\left(1-\tau_{s}\right)^{n-2} .
\end{aligned}
$$

Note that if there is only one failure among $n-1$ nodes, then node $t x$ receives a successful RTS setting the NAV for as long as at least one DATA transmission. $P_{\text {coll }}$, the probability that the node sets its NAV for a short duration that contains no DATA reception, given that the node is carrier sensing with zero NAV, is given by $P_{\text {coll }}=1-P_{\text {idle }}-P_{\text {succ }}$.

\subsection{Probability of Collision: $p$}

An RTS or CTS packet that is not received correctly by neighbors inside the carrier sensing range may cause collision of frames other than RTS frames due to hidden terminals in multihop networks. In this paper, collisions among RTS, CTS, DATA, or ACK frames with each other are also considered in the calculation of probability of collision. Let us denote the $\sigma$ duration prior to RTS transmission from $t x \rightarrow r x$ with $\Delta_{0}$, the first $\sigma$ portion of RTS transmission with $\Delta_{1}$, and the time duration afterwards up to the first $\sigma$ portion of the CTS frame with $\Delta_{2}$ as 


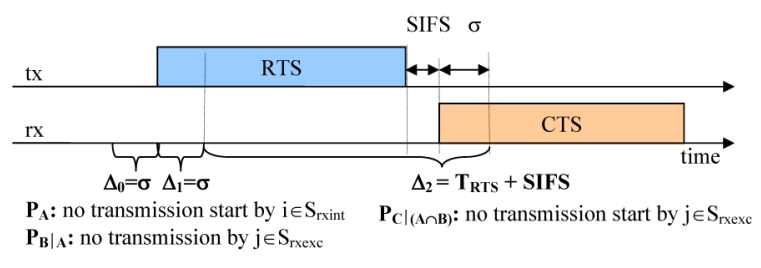

Fig. 4. Illustration of calculation of $p$.

illustrated in Fig. 4. An RTS/CTS transmission from $t x \rightarrow$ $r x$ is successful if and only if all the Events $A, B$, and $C$ take place, given that a transmission occurs:

1. Event $A$. No node $i \in S_{\text {rxint }}-\{t x\}$ starts a transmission during $\Delta_{0}$ and $\Delta_{1}$. The probability of Event $A$ corresponds to $P_{A}=\prod_{k=0}^{1}\left(1-\tau_{A_{k}}\right)^{\text {Nrxint }-1}$, where $\tau_{A_{0}}$ is the probability that node $i \in S_{\text {rxint }}-\{t x\}$ starts a transmission during $\Delta_{0}$ and $\tau_{A_{1}}$ is the probability that node $i \in S_{\text {rxint }}-\{t x\}$ starts a transmission during $\Delta_{1}$.

2. Event $B$. No node $j \in S_{\text {rxexc }}$ is transmitting during $\Delta_{1}$. The probability of Event $B$ given that Event $A$ occurs is denoted by $P_{B \mid A}$ and is calculated by $P_{B \mid A}=\left(1-\tau_{B}\right)^{N_{\text {rrexc }}}$, where $\tau_{B}$ is the probability that node $j \in S_{\text {rxexc }}$ is transmitting during $\Delta_{1}$, given that Event $A$ occurs.

3. Event $C$. No node $j \in S_{\text {rxexc }}$ starts a transmission during $\Delta_{2}$. The probability of Event $C$ given that Events $A$ and $B$ occur is denoted by $P_{C \mid(A \cap B)}$ and corresponds to the probability $P_{C \mid(A \cap B)}=\left(1-\tau_{C}\right)^{\text {Nrxexc } \frac{\Delta_{2}}{\sigma}}$, where $\tau_{C}$ is the probability that node $j \in S_{\text {rxexc }}$ starts a transmission during $\Delta_{2}$, given that Events $A$ and $B$ occur.

The probability of collision is given by

$p=1-\left\{\left(1-\tau_{A_{0}}\right)\left(1-\tau_{A_{1}}\right)\right\}^{N_{\text {rxint }}-1}\left\{\left(1-\tau_{B}\right)\left(1-\tau_{C}\right)^{\frac{\Delta_{2}}{\sigma}}\right\}^{N_{\text {rxxexc }}}$.

The calculation of $\tau_{A_{0}}, \tau_{A_{1}}, \tau_{B}$, and $\tau_{C}$ is based on the fixed length time slot notion and is given by

$$
\tau_{i}=\frac{\pi_{\text {transmit }}^{i}}{\pi_{\text {allowed }}^{i}}, \text { for } i=\left\{A_{0}, A_{1}, B, C\right\},
$$

where $\pi_{\text {transmit }}^{i}$ corresponds to the sum of steady state probabilities of states where nodes have the opportunity to transmit and $\pi_{\text {allowed }}^{i}$ corresponds to the sum of steady state probabilities of allowed states for $i=\left\{A_{0}, A_{1}, B, C\right\}$ and are given by (the derivation of these probabilities is given in Appendix B),

$$
\begin{aligned}
\pi_{\text {transmit }}^{A_{0}}= & \pi_{t c} \frac{\sigma}{T_{t c}}+K_{A} \pi_{r s} \frac{\sigma}{T_{t s}} \\
\pi_{\text {allowed }}^{A_{0}}= & 1-\pi_{t s}-\pi_{t c} \frac{T_{t c}-2 \sigma}{T_{t c}}-K_{A} \pi_{r s} \frac{T_{t s}-2 \sigma}{T_{t s}} \\
& -R_{t x \text { Srxint }}\left(\pi_{r s} \frac{T_{r s}-2 \sigma}{T_{r s}}+\pi_{r c} \frac{T_{r c}-2 \sigma}{T_{r c}}\right) \\
& -R_{\text {int Srxint }}\left(\pi_{r s} \frac{T_{r s}-2 \sigma}{T_{r s}}+\pi_{r c} \frac{T_{r c}-2 \sigma}{T_{r c}}\right)
\end{aligned}
$$

$$
\begin{aligned}
& \pi_{\text {transmit }}^{A_{1}}=\pi_{t c} \frac{\sigma}{T_{t c}}+K_{A} \pi_{r s} \frac{\sigma}{T_{t s}}, \\
& \pi_{\text {allowed }}^{A_{1}}=1-\pi_{\text {idle }}-\pi_{t s}-\pi_{t c} \frac{T_{t c}-\sigma}{T_{t c}}-K_{A} \pi_{r s} \frac{T_{t s}-\sigma}{T_{t s}} \\
& -R_{t x \text { Srxint }}\left(\pi_{r s} \frac{T_{r s}-\sigma}{T_{r s}}+\pi_{r c} \frac{T_{r c}-\sigma}{T_{r c}}\right) \\
& -R_{\text {intSrxint }}\left(\pi_{r s} \frac{T_{r s}-\sigma}{T_{r s}}+\pi_{r c} \frac{T_{r c}-\sigma}{T_{r c}}\right) \\
& \pi_{\text {transmit }}^{B}=\pi_{t s} \frac{T_{t s}-D I F S}{T_{t s}}+\pi_{t c} \frac{T_{R T S}}{T_{t c}} \\
& +K_{B} \pi_{r s} \frac{T_{C T S}+T_{D A T A}+T_{A C K}+2 S I F S}{T_{t s}}, \\
& \pi_{\text {allowed }}^{B}=1-R_{\text {intSrxexc }}\left(\pi_{r s}+\pi_{r c}\right) \\
& -R_{t x \operatorname{Srxexc}}\left(\pi_{r s} \frac{T_{r s}-\sigma}{T_{r s}}+\pi_{r c} \frac{T_{r s}-\sigma}{T_{r s}}\right) \\
& -R_{r x \operatorname{Srxexc}}\left(\pi_{r s} \frac{T_{r s}-D I F S}{T_{r s}}-\pi_{r c} \frac{T_{r c}-E I F S}{T_{r c}}\right), \\
& \pi_{\text {transmit }}^{C}=\pi_{t s} \frac{\sigma}{T_{t s}}+\pi_{t c} \frac{\sigma}{T_{t c}}+K_{B} \pi_{r s} \frac{\sigma}{T_{t s}}, \\
& \pi_{\text {allowed }}^{C}=1-\pi_{t s} \frac{T_{t s}-\sigma-D I F S}{T_{t s}}-\pi_{t c} \frac{T_{R T S}-\sigma}{T_{t c}} \\
& -K_{B} \pi_{r s} \frac{T_{t s}-T_{R T S}-S I F S-\sigma-D I F S}{T_{t s}} \\
& -\left(1-R_{\text {excSrxexc }}\right)\left(\pi_{r s}+\pi_{r c}\right) .
\end{aligned}
$$

$K_{A}$ represents the fraction of successful receptions that are destined to node $i \in S_{\text {rxint }}-\{t x\}$ from a transmitter $\in S_{e x c}^{t x \rightarrow i}$ and is calculated by averaging over all $i \in S_{\text {rxint }}-\{t x\}$; whereas $K_{B}$ represents the fraction of successful receptions that are destined to node $j \in S_{\text {rxexc }}$ from a transmitter $\epsilon$ $S_{e x c}^{r x \rightarrow j}$ and is calculated by averaging over all $j \in S_{r x e x c}$. The reader is referred to [26] for the derivation of $K_{1}, K_{A}$, and $K_{B}$.

\section{ENERgy MOdel for IEEE 802.11 DCF-BASEd MuLTIHOP Ad HOC NeTWORKS}

In this section, we propose an energy model which finds out the energy consumption for successfully delivering one bit of DATA to its destination in a IEEE 802.11 based multihop network considering carrier sensing, collisions, freezing mechanism in backoff and energy consumption due to overhearing. The total energy cost of transmitting one successful bit over a path is called Energy Per Bit (EPB) and is given by

$$
E P B=E_{t x}+E_{r x}+E_{\text {overhear }},
$$

where $E_{t x}\left(E_{r x}\right)$ is the total energy per bit consumed by all path nodes for transmitting (receiving), $E_{\text {overhear }}$ is the total energy per bit consumed by all path and neighbor nodes while overhearing, i.e., receiving packets intended for other nodes. Path nodes are the source and destination nodes plus any relay nodes in between. Neighboring nodes are the nodes inside the union of transmission areas of all path nodes, excluding the path nodes. The path nodes consume energy while transmitting/receiving and overhearing; and the neighbor nodes consume energy while overhearing. EPB is calculated such that energy spent for transmit and receive is taken into account while energy spent during idle 
mode is excluded. For the computation of the idle energy the reader is referred to [26].

We assume that the PHY layer may be in transmit, receive, or idle mode (neglecting sleep mode) and denote the power consumed by $P w r_{t x}, P w r_{r x}$, and $P w r_{i d l e}$, respectively. EPB is calculated by considering the energy consumed by a DATA packet and any related control packets, collisions, retransmissions, and packet drops due to that specific DATA packet. For a single successful DATA packet to be received by the destination, a total of $N_{\text {succ }}$ successful transmissions and $N_{\text {drop }}$ packet drops take place on the average, over an $h$-hop path. $N_{\text {succ }}$ and $N_{\text {drop }}$ are obtained by averaging the total number of successful/failed transmissions over all $h$-hop paths $\gamma$ consisting of nodes $\left\{x_{0}, x_{1}, \ldots, x_{h}\right\}$, where the averaging function over all paths is denoted by $a v g_{\gamma}$ :

$$
\begin{aligned}
& N_{\text {succ }}=\operatorname{avg}_{\gamma}\left\{\sum_{i=0}^{h-1}\left(\left(1-p^{M}\right)^{i} \prod_{j=1}^{i}\left(1-P_{i f q}\left(x_{h-j}\right)\right)\right)^{-1}\right\}, \\
& N_{\text {drop }}=N_{\text {succ }} \frac{p^{M}}{\left(1-p^{M}\right)} .
\end{aligned}
$$

Let us denote the duration where transmit or receive energy is spent over a link for one successful transmission by $T_{\text {succ }}$ and for a dropped packet by $T_{d r o p}$ :

$$
\begin{aligned}
& T_{\text {succ }}=\bar{n}_{M} T_{R T S}+T_{C T S}+T_{D A T A}+T_{A C K}, \\
& T_{\text {drop }}=M T_{\text {RTS }} .
\end{aligned}
$$

$\bar{n}_{M}$ is the average number of dropped packets due to exceeding $M$ and is calculated as follows:

$$
\bar{n}_{M}=\sum_{i=0}^{M-1} i p^{i}(1-p)+M p^{M} .
$$

$T_{\text {busy }}$ denotes the total time duration where transmit or receive energy is spent over an $h$-hop path

$$
T_{\text {busy }}=N_{\text {succ }} T_{\text {succ }}+N_{\text {drop }} T_{\text {drop }} \text {. }
$$

$E_{t x}$ and $E_{r x}$ are given by

$$
\begin{aligned}
E_{t x} & =\frac{1}{b_{D A T A}}\left(P w r_{t x}-P w r_{i d l e}\right) T_{b u s y}, \\
E_{r x} & =\frac{1}{b_{D A T A}}\left(P w r_{r x}-P w r_{i d l e}\right) T_{b u s y} .
\end{aligned}
$$

A specific transmission flowing through an $h$-hop path consumes no additional energy at an overhearing node if the NAV of the overhearing node is already set. Recalling that $P_{\text {idle }}$ is a conditional probability conditioned on the event that the node is carrier sensing with zero NAV, and noting that the probability that a node is carrier sensing with zero NAV is $1-\tau$, the unconditional probability that NAV of a node is not set becomes $(1-\tau) P_{\text {idle }}$. Since the number of overhearing nodes is $n-2$, excluding the transmitter and receiver, we obtain

$$
E_{\text {overhear }}=\frac{(n-2)(1-\tau) P_{\text {idle }}\left(P w r_{r x}-P w r_{i d l e}\right) T_{\text {busy }}}{b_{D A T A}} .
$$

Our extensive numerical studies show that the accuracy of the analytical model in predicting EPB degrades as the
TABLE 2

Parameters Used for Both the Analytical Model and Simulation Runs

\begin{tabular}{l|l}
\hline Data rate & $11 \mathrm{Mbps}$ \\
Basic rate & $1 \mathrm{Mbps}$ \\
PLCP rate & $1 \mathrm{Mbps}$ \\
\hline$W_{0}$ & 32 for random, 128 for \\
& hexagonal topology \\
B & 3 \\
Short retry count & 7 \\
Long retry count & 3 \\
\hline SlotTime & $20 \mu$ s \\
DATA & 1072 bytes \\
RTS & 44 bytes \\
CTS & 44 bytes \\
ACK & 44 bytes \\
SIFS & $10 \mu$ s \\
DIFS & $50 \mu \mathrm{s}$ \\
EIFS & $412 \mu \mathrm{s}$ \\
\hline IFQ buffer size & 5 \\
\hline RxSensitivity & $-70 \mathrm{dBm}$ \\
path loss exponent $(\eta)$ & 3 \\
$P w r_{t x}$ & $1.425+0.25 h^{-n} \mathrm{~W}$ \\
$P w r_{r x}$ & $1.425 \mathrm{~W}$ \\
$P w r_{i d l e}$ & $1.319 \mathrm{~W}$ \\
\hline
\end{tabular}

topology and traffic pattern become more irregular due to the averaging of $p$. However, the general characteristics of the EPB curves and the implications regarding relaying are not affected.

\section{Numerical Results}

Energy efficiency of relaying strategies are studied for different topologies deployed in a fixed area: a hexagonally placed 469-node regular topology with $h=\{1,2,3,6\}$ and 12 randomly generated topologies 5 with 50 , 4 with 100 , and 3 with 200 nodes with $h=\{1,3\}$ are compared through analysis and simulations. For the hexagonal topology, source-destination pairs are chosen so that all possible linear paths carry traffic, while for the random topologies all source-destination pairs that have a three-hop path in between are chosen. The hexagonal topology is homogeneous in topology and traffic distribution, whereas the random topologies have no homogeneity. The simulations are conducted using Network Simulator 2, version nsallinone-2.34 [28]. The parameters used for both the analytical model and the simulations are listed in Table 2. The power consumption values of transmit, receive, and idle modes of the $2.4 \mathrm{GHz}$ IEEE 802.11b Wavelan card are adapted [29]. Fixed point iterations are carried with a precision of $10^{-10}$.

\subsection{Total EPB}

We first assume that nodes have perfect sleeping mechanisms and hence the energy spent in idle mode is neglected. The average EPBs computed by the analytical model and simulations for random topologies of size 50,100, and 200 nodes are plotted in Fig. 5, as a function of the average packet generation rate $\lambda_{o}$. The results show that the energy model is quite well in predicting the EPB for random wireless multihop networks. The model predicts the energy 


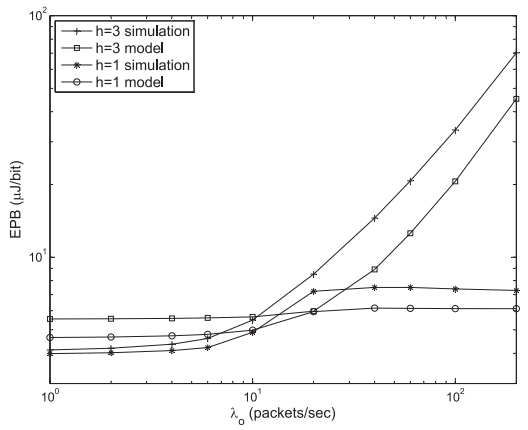

(a)

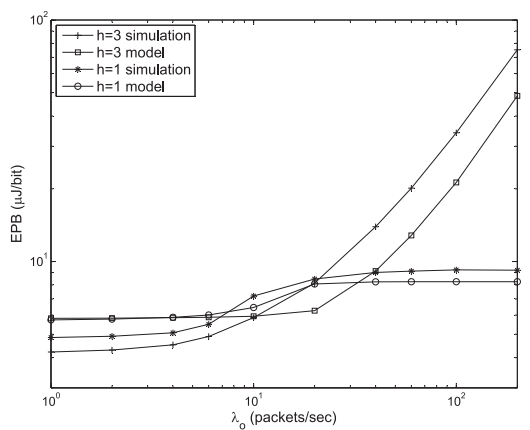

(b)

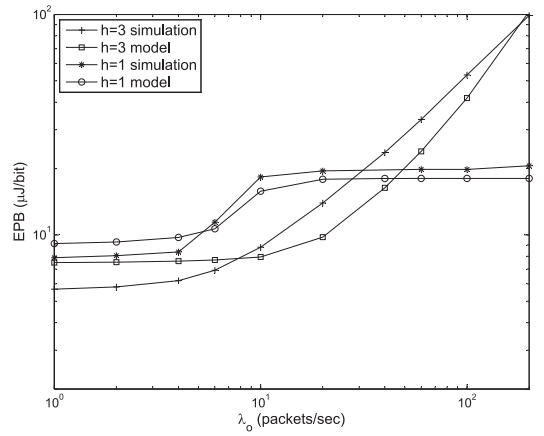

(c)

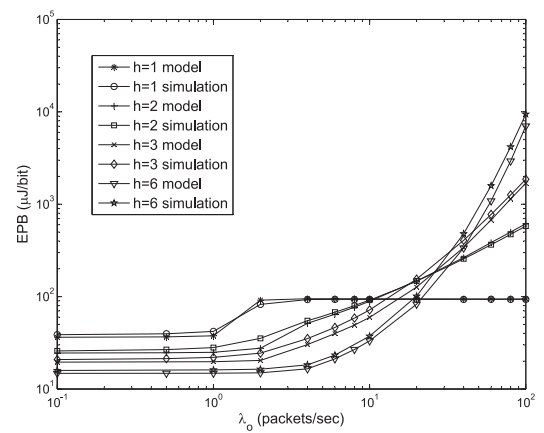

(d)

Fig. 5. EPB obtained from anaytical model and simulations without inclusion of energy consumed in the idle mode for (a) 50-node random, (b) 100-node random, (c) 200-node random, and (d) 469-node hexagonal topologies.

consumption fairly well for a wide range of traffic loads. The error observed for random topologies stems from computation of average values for $p$ and geometry related variables in order to come up with a computationally tractable analytical model. As the number of nodes in the network increases, more energy-efficient three-hop-path alternatives emerge and EPB difference between $h=1$ and

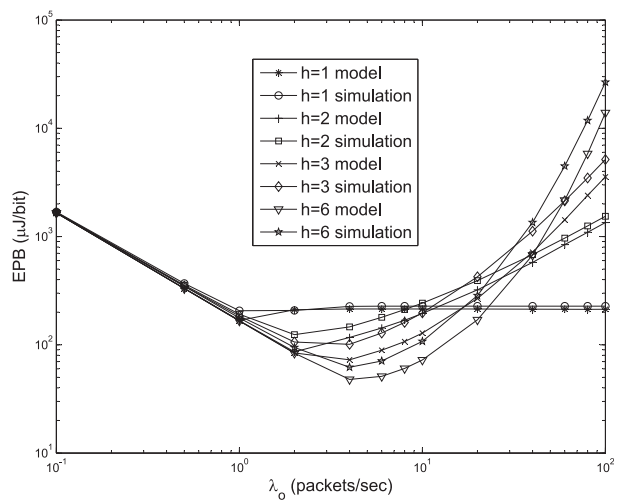

Fig. 6. EPB obtained from the analytical model and simulations for the hexagonal topology with $h=\{1,2,3,6\}$ with inclusion of energy consumed in the idle mode.

$h=3$ increases under moderate traffic. At heavy traffic load, EPB with multihopping increases sharply due to heavy collisions and increased number of retransmissions, and high offered traffic load make the network unstable.

It is observed that EPB increases as the node density is increased due to increased receive energy consumption by increased number of overhearing nodes. Furthermore, multihopping $(h=3)$ becomes more energy efficient than direct transmission $(h=1)$ at moderate traffic loads as the number of nodes and hence the density increases. This is due to the fact that denser networks allow more energyefficient multihop paths.

Since the numerical solution of the analytical model requires substantially long computation times, we used an hexagonal topology with 469 nodes in order to study larger networks since the symmetric nature of this topology simplifies calculations. EPB computed by the analytical model and simulations for the hexagonal topology as a function of the packet generation rate $\lambda_{o}$ are shown in Fig. $5 \mathrm{~d}$. The error is less compared to random topologies due to the homogeneity of the hexagonal topology and traffic. It is observed that multihopping is significantly more energy efficient than direct transmission under light-to-moderate traffic for the dense and regular hexagonal topology. More discernible energy savings with $h=6$ is due to the availability of many multihop paths with equal hop lengths.

Previous studies on minimum-energy routing consider solely the energy consumption at the amplifier due to transmissions and it is stated in these studies that multihop paths are more energy efficient [1], [2]. Based on our results, this statement is valid only for low-to-moderate traffic loads and for dense topologies where more energy-efficient alternative multihop paths exist. Consideration of MAC contention changes the optimal relaying strategy to direct transmission at heavy traffic loads, reducing the energy consumption by $400-500$ percent for random topologies and by 2-orders of magnitude for the hexagonal topology and the energy saving increases as the traffic gets heavier.

\subsection{Effect of Idle Energy and Sleeping Mechanism}

EPB with the inclusion of power consumption in the idle mode is plotted in Fig. 6 for the hexagonal topology, which corresponds to the energy consumption where the transceiver never enters the sleep mode. Consideration of idle 


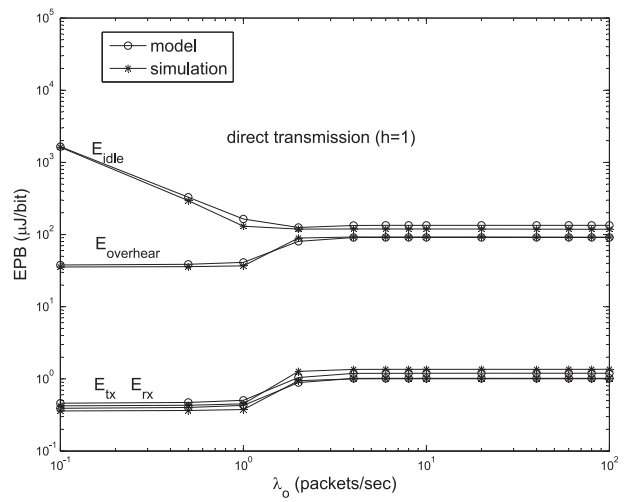

(a)

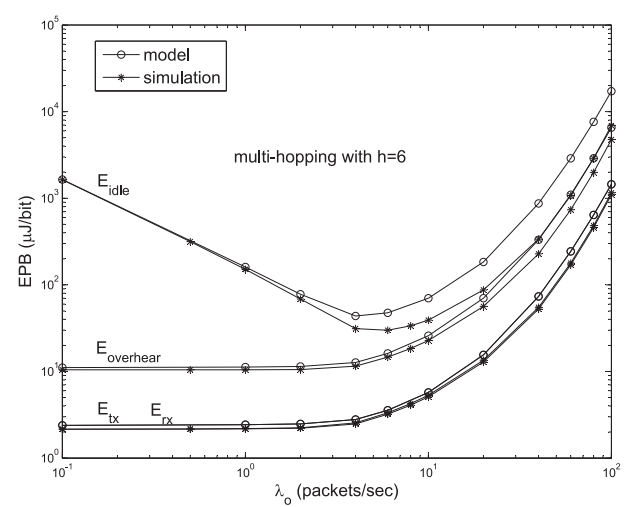

(b)

Fig. 7. Idle, overhear, transmit, and receive energies per bit for (a) direct transmission and (b) multihopping with $h=6$.

energy makes any relaying strategy equivalently energy efficient for light traffic loads. For moderate and heavy traffic loads, EPB exhibits a similar behavior as the case of perfect sleep management shown in Fig. 5d.

\subsection{Components of EPB}

The components of EPB, namely, idle, overhear, transmit, and receive energies per bit for the hexagonal topology are shown in Fig. 7 for direct transmission $(h=1)$ and for multihopping with $h=6$ as the traffic load changes. It is observed that idle and receive energy during overhearing are responsible for most of the energy consumed, especially at light traffic loads. The increase in all components with multihopping under heavy traffic is due to the increased packet drops at intermediate hops and hence the increased time per bit. Energy spent during transmission and reception at the intended receiver constitute a small portion of EPB, about 1 percent of EPB for direct transmission and about 10 percent of EPB for $h=6$ under moderate-to-heavy traffic. It is seen that inclusion of energy expenditure during idle listening, carrier sensing, collisions, freezing mechanism in backoff and extra energy consumption due to overhearing significantly affect the energy consumption.

\subsection{Effect of Processing Power}

The EPB with the inclusion of energy consumption in idle mode versus processing power at relay nodes is plotted in Fig. 8 for different relaying strategies using the hexagonal topology and $\lambda_{o}=\{0.5,4,60\}$ packets $/ \mathrm{sec}$. The results show that the processing power affects the optimal hop number

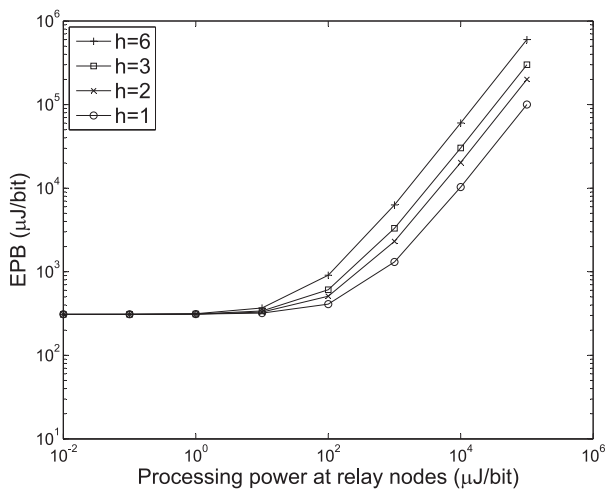

(a)

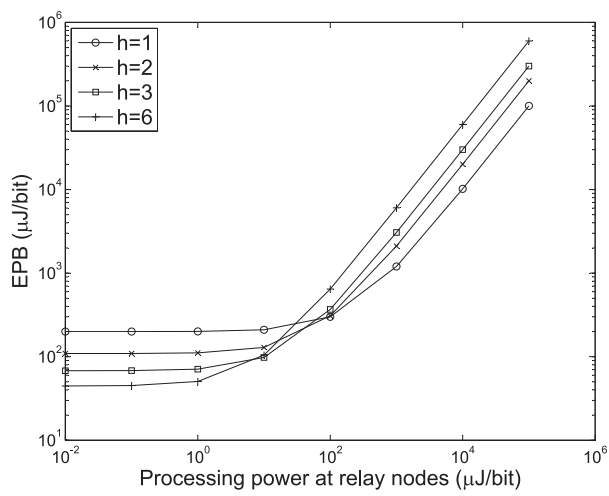

(b)

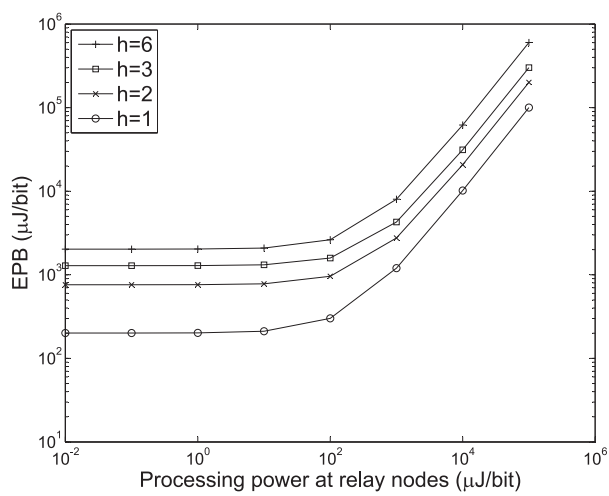

(c)

Fig. 8. EPB with idle energy versus processing power for (a) $\lambda_{o}=0.5$, (b) $\lambda_{o}=4$, and (c) $\lambda_{o}=60$ packets $/ \mathrm{sec}$

for energy efficient relaying only under moderate traffic loads. Under moderate traffic loads, $h=6$ is optimum under low processing power, whereas $h=1$ becomes more energy efficient as processing power increases. Meanwhile, under light and heavy traffic $h=1$ is the most energy efficient independent of the processing power.

\subsection{Effect of Contention Window Size}

EPB with idle energy consumption versus minimum contention window size, $W_{0}=\{32,64,128\}$, for $h=\{1,2,3,6\}$, and $\lambda_{o}=\{0.5,4,60\}$ packets $/ \mathrm{sec}$ for the hexagonal topology are shown in Fig. 9. EPB is observed to be independent of the minimum contention window size under light traffic loads. Under heavy traffic, increasing the contention window decreases EPB by about up to 50 percent for $h=\{1,2,3\}$ and by about 8 percent for $h=6$. This result reveals that 


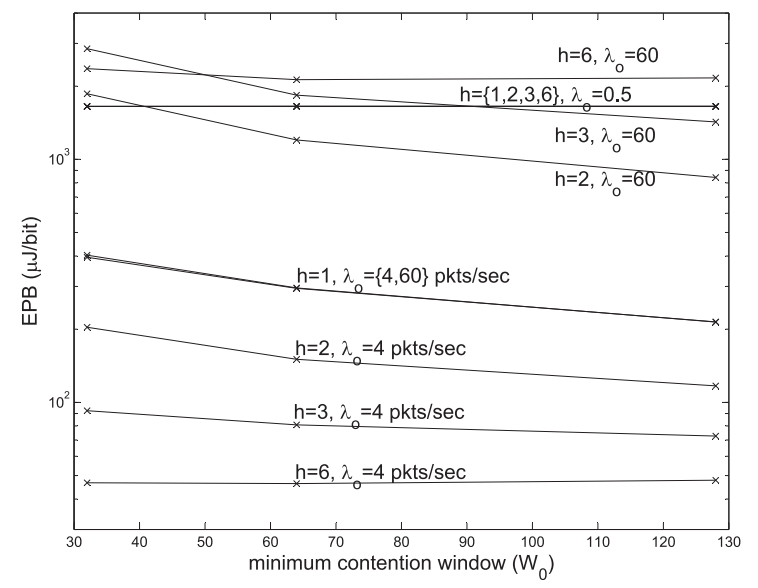

Fig. 9. EPB with idle energy consumption versus minimum contention window $W_{0}$, for $h=\{1,2,3,6\}$ and $\lambda_{o}=\{0.5,4,60\}$ packets/sec.

controlling the contention window size is a successful crosslayered energy-efficiency approach, especially under heavy traffic.

\section{Conclusions}

An analytical model for IEEE 802.11 DCF and an energy model representing the energy consumption per bit in an IEEE 802.11 DCF based wireless multihop network are developed for investigating the effect of relaying on energy efficiency. It is shown that the analytical model is accurate in predicting the energy efficiency over a wide range of scenarios. The results show that the energy-efficient routing strategy depends not only on the processing power as shown before [4], [5], [6], [7], but also on the traffic load. It is shown that the dependence on processing power is valid only for a specific range of traffic loads. Previous studies, e.g., [1], [2], that consider solely the energy consumption due to transmissions, state that multihop paths are more energy efficient. Our results show that this is valid for lowto-moderate traffic loads with a perfect sleeping regime or for moderate traffic loads with no sleeping regime and dense topologies with more energy efficient alternative multi hop paths.

Transmission power reduction with multi hopping decreases the number of contending stations in the transmission range, which results in less collisions, retransmissions, backoff, and freezing mechanisms at the MAC layer, reducing the energy consumption of multihopping for low-to-moderate traffic loads. However, as the traffic increases, MAC layer contentions increase and end-to-end throughput approaches to zero due to heavy packet drops at intermediate hops, resulting in an increase in the energy per bit. It is shown through simulations and analytical model that multihopping becomes more energy efficient up to some traffic rate and direct transmission becomes more energy efficient afterwards. Furthermore, the advantage of multihopping is larger for dense and regular topologies. The results show that the energy consumed per bit by direct transmission is more robust in a multihop network, because excessive packets are dropped at the interface queues before being transmitted. But in multihop routing, packet drops

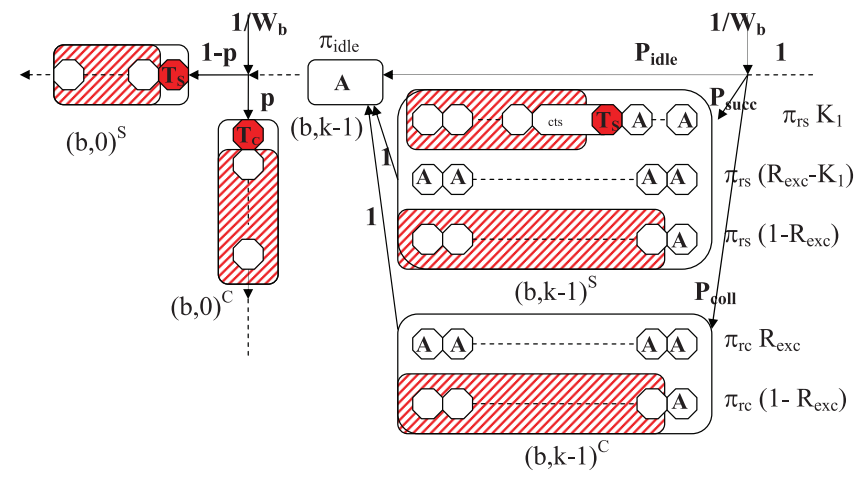

Fig. 10. Illustration of calculation of NAV setting probabilities based on fixed-slot notion.

occur at the wireless links, substantially increasing the energy waste especially when the network is congested.

The results indicate that a cross-layered relaying approach, which takes energy expenditure due to MAC contentions into account and dynamically changes the relaying strategy according to the network traffic load, can substantially save energy in a realistic wireless multihop network where the network traffic load changes in time. As a future work, an adaptive cross-layered relaying strategy will be investigated.

\section{ApPendix A}

\section{Derivation of NaV Setting Probabilities}

The NAV setting probabilities are functions of the variables $\tau_{s}, \tau_{c}$, and $\pi_{\text {allowed }}^{N A V}$, that are given in (5) and that are derived based on the fixed length slot notion, so that the states of the SMC are divided into states of residence times of $\sigma$ transforming to a DTMC. A portion of the obtained DTMC that is used in calculation of NAV setting probabilities is shown in Fig. 10.

The states at which a successful transmission is started are indicated by $T_{S}$-labeled states in Fig. 10. A successful transmission is started by a node $i \in S_{t x}-\{t x\}$ during the first $\sigma$ duration of transmit success states, corresponding to the steady-state probability $\pi_{t s} \frac{\sigma}{T_{t s}}$, and during the first $\sigma$ duration of a specific portion of receive success states, where a CTS reply to a node $j \in S_{e x c}^{t x \rightarrow i}$ is started, corresponding to $K_{1} \pi_{r s} \frac{\sigma}{T_{t s}}$. The multiplicand $K_{1}$ gives the fraction of successful receptions from nodes inside $S_{e x c}^{t x \rightarrow i}$ that are destined to node $i$ only. Hence, the steady-state probability of the $T_{S}$-labeled states constitutes the numerator of $\tau_{s}$ given in (5). The state at which a collision is started is indicated by the $T_{C}$-labeled state in Fig. 10, corresponding to the first $\sigma$ duration of transmit collision states. The steady-state probability of the $T_{C}$-labeled state, $\pi_{t c} \frac{\sigma}{T_{t c}}$, gives the numerator of $\tau_{c}$.

The $A$-labeled states shown in Fig. 10 together with the $T_{S}$-labeled and $T_{C}$-labeled states are the states that node $i \in S_{t x}-\{t x\}$ is allowed to be at while carrier sensing with zero NAV. The sum of the steady state probabilities of these states corresponds to $\pi_{\text {allowed }}^{N A V}$. Note that nodes are not allowed to transmit anything, and receive a successful transmission or a collision from any node $j \in S_{t x}-\{t x\}$, 
excluding the first $\sigma$ duration of receive success and receive collision states.

\section{APPENDIX B \\ DeRIVATION OF $p$}

The probability of collision given that a transmission occurs from $t x \rightarrow r x$, is a function of $\tau_{A_{0}}, \tau_{A_{1}}, \tau_{B}$, and $\tau_{C}$, as given in (6). These variables are also derived based on the fixed length slot notion, so that the states of the SMC are divided into states of residence times of $\sigma$ transforming to a DTMC. A portion of the obtained DTMC that is used to illustrate the calculation of $\tau_{A_{0}}, \tau_{A_{1}}, \tau_{B}$, and $\tau_{C}$ is shown in Fig. 11. $T$-labeled states form the numerator $\tau_{\text {transmit }}^{i}$, whereas $T$-labeled and $A$-labeled states together form the denominator of $\tau^{i}$, for $i=A_{0}, A_{1}, B, C$. The $T$-labeled states correspond to the states where the concerned nodes have the opportunity to transmit, whereas the $A$-labeled states correspond to the states where the concerned nodes are allowed to be in during the concerned time interval.

$\tau_{A_{0}}$ is the probability that node $i \in S_{\text {rxint }}-\{t x\}$ starts a transmission during $\Delta_{0}$. The numerator of $\tau_{A_{0}}, \pi_{\text {transmit }}^{A_{0}}$ corresponds to the sum of steady state probabilities of states where node $i \in S_{\text {rxint }}$ during $\Delta_{0}$ has the opportunity to transmit. These are the states where the first $\sigma$ portion of a collided RTS is sent and the first $\sigma$ portion of a CTS response to a transmitter $k \in S_{e x c}^{t x \rightarrow i}$ is sent. $\pi_{\text {transmit }}^{A_{0}}$ is calculated by summing up the steady state probabilities of the $T$-labeled states shown in Fig. 11a. The $A$-labeled states plus the $T$-labeled states give us the allowed states, the sum of steady state probabilities of which corresponds to $\pi_{\text {allowed }}^{A_{0}}$. There are certain states that node $i$ cannot be in during $\Delta_{0}$ since $p$ is conditioned on occurrence of a transmission from $t x \rightarrow r x$. For example, node $i$ cannot be in transmit success states, since any transmission of node $i$ during $\Delta_{0}$, given that a transmission from $t x \rightarrow r x$ starts during $\Delta_{1}$, would be a collision. Also, node $i$ cannot be in transmit collision state, excluding the first and last $\sigma$ duration of a collision, since node $i$ being in these states would suppress the $t x \rightarrow r x$ transmission. Likewise, node $i$ cannot receive from nodes inside $S_{t x \text { Srxint }}$ and $S_{\text {intSrxint }}$, excluding the first and last $\sigma$ duration of receive success and receive collision states, for the same reasoning.

$\tau_{A_{1}}$ is the probability that node $i \in S_{\text {rxint }}-\{t x\}$ starts a transmission during $\Delta_{1}$. The A-labeled and $T$-labeled states used in calculation of $\pi_{\text {transmit }}^{A_{1}}$ and $\pi_{\text {allowed }}^{A_{1}}$ are shown in Fig. $11 \mathrm{~b}$. Some allowed states during $\Delta_{0}$ shown in Fig. 11a are not allowed during $\Delta_{1}$. For example, node $i \in S_{\text {rxint }}$ is not allowed to be in idle states, since it receives the transmission from $t x \rightarrow r x$ during $\Delta_{1}$. Also, node $i$ cannot be in the last $\sigma$ duration of transmit collision or receive states since node $i$ being in these states would suppress the $t x \rightarrow r x$ transmission.

$\tau_{B}$ is the probability that node $j \in S_{\text {rxexc }}$ is transmitting (i.e., making a successful transmission or collision, or making a successful reception destined to itself) during $\Delta_{1}$, given Event $A$, i.e., nodes $\in S_{\text {rxint }}-\{t x\}$ do not start a transmission. The $T$-labeled states and the $A$-labeled states for nodes in $S_{\text {rxexc }}$ during $\Delta_{1}$ are shown in Fig. 11c. Node $j \in S_{\text {rxexc }}$ is allowed to be transmitting a successful transmission, a collision to any node or a CTS response to

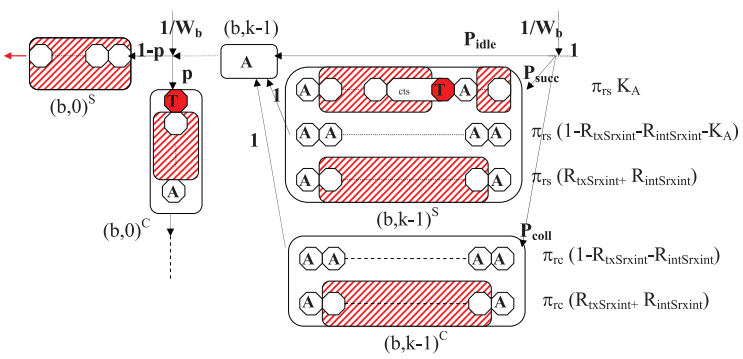

(a)

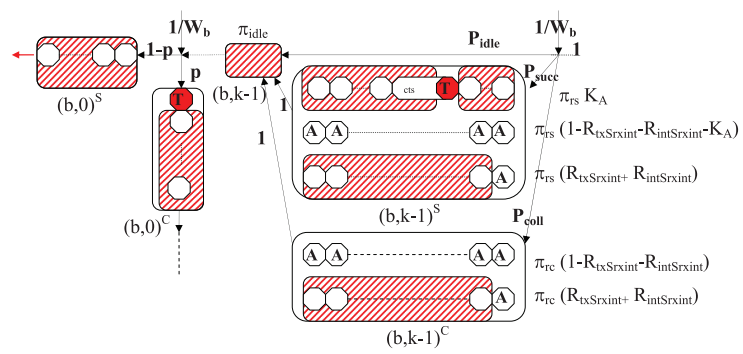

(b)

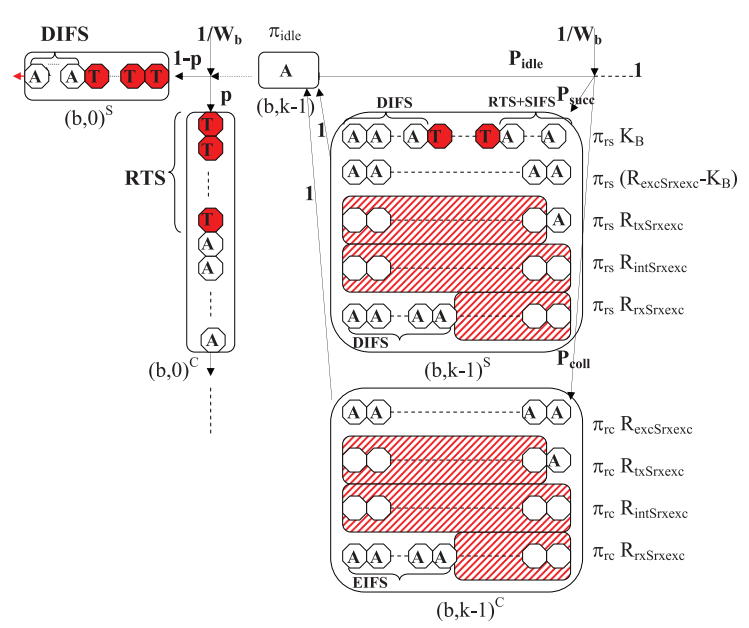

(c)

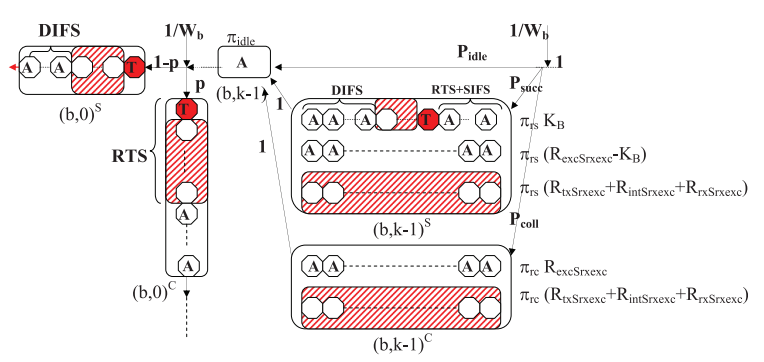

(d)

Fig. 11. Illustration of transmit ( $T$-labeled) and allowed ( $A$-labeled) states for (a) node $i \in S_{\text {rxint }}-\{t x\}$ during $\Delta_{0}$ for calculation of $\tau^{A_{0}}$, (b) node $i \in S_{\text {rxint }}-\{t x\}$ during $\Delta_{1}$ for calculation of $\tau^{A_{1}}$, (c) node $j \in$ $S_{\text {rxexc }}$ during $\Delta_{1}$ for calculation of $\tau^{B}$, and (d) node $j \in S_{\text {rxexc }}$ during $\Delta_{2}$ for calculation of $\tau^{C}$.

some node $k \in S_{\text {rxSrxexc }} \bigcup S_{\text {excSrxexc }}$, since node $j \in S_{\text {rxexc }}$ is not exposed to $t x$ carrier. Node $j$ is not allowed to be in receive states from nodes inside $S_{t x \text { Srxexc }}$, except the first $\sigma$ portion, since a transmission is perceived after $\sigma$ duration by the PHY layer. Node $j$ is not allowed to receive from $S_{\text {intSrxexc }}$ due to conditioning of occurrence of Event $A$. Node $j$ cannot receive from nodes inside $S_{r x \text { Srxexc }}$ during 
the busy periods of received transmissions, i.e., $T_{r s}-D I F S$ and $T_{r c}-E I F S$, since this implies that some node inside $S_{\text {rxexc }}$ is transmitting where this probability is already captured by taking the power $N_{\text {rxexc }}$ of $1-\tau_{B}$.

$\tau_{C}$ is the probability that node $j \in S_{\text {rxexc }}$ starts a transmission, given that Events $A$ and $B$ occur. The $T$-labeled states and the $A$-labeled states for nodes in $S_{\text {rxexc }}$ during $\Delta_{2}$ are shown in Fig. 11d. Node $j \in S_{\text {rxexc }}$ cannot be transmitting a successful transmission, a collision or a CTS response to some node $k \in S_{\text {excSrxexc }}$, except the first $\sigma$ portion, due to condition on occurrence of Event $B$. Node $j$ cannot receive from nodes inside $S_{t x \text { Srxexc }}$ during $\Delta_{2}$ since these nodes are silenced already by the transmission from $t x \rightarrow r x$. Node $j$ cannot receive from nodes inside $S_{\text {intSrxexc }}$ due to condition on the Event $A$. Node $j$ cannot receive from nodes inside $S_{\text {rxSrxexc }}$ due to condition on the Event $B$.

\section{REFERENCES}

[1] S. Singh, M. Woo, and C.S. Raghavendra, "Power-Aware Routing in Mobile Ad Hoc Networks," Proc. Fourth Ann. ACM/IEEE Int'l Conf. Mobile Computing and Networking, pp. 181-190, Oct. 1998.

[2] K. Scott and N. Bambos, "Routing and Channel Assignment for Low Power Transmission in PCS," Proc. Fifth IEEE Int'l Conf. Universal Personal Comm. (lCUPC'96), vol. 2, pp. 498-502, Oct. 1996.

[3] P. Agrawal, "Energy Efficient Protocols for Wireless Systems," Proc. Ninth IEEE Int'l Symp. Personal, Indoor and Mobile Radio Comm. (PIMRC '98), vol. 2, pp. 564-569, Sept. 1998.

[4] P. Chen, B. O'Dea, and E. Callaway, "Energy Efficient System Design with Optimum Transmission Range for Wireless Ad Hoc Networks," Proc. IEEE Int'l Conf. Comm. (ICC '02), vol. 2, pp. 945952, May 2002.

[5] Z. Shelby, C. Pomalaza-Raez, and J. Haapola, "Energy Optimization in Multihop Wireless Embedded and Sensor Networks," Proc. 15th IEEE Int'l Symp. Personal, Indoor, and Mobile Radio Comm. (PIMRC '04), Sept. 2004.

[6] E.I. Oyman and C. Ersoy, "Overhead Energy Considerations for Efficient Routing in Wireless Sensor Networks," Computer Networks, vol. 46, no. 4, pp. 465-478, Nov. 2004.

[7] W.R. Heinzelman, A. Chandrakasan, and H. Balakrishnan, "Energy-Efficient Communication Protocol for Wireless Microsensor Networks," Proc. 33rd Int'l Conf. System Sciences, pp. 1-10, Jan. 2000.

[8] U.C. Kozat, I. Koutsopoulos, and L. Tassiulas, "A Framework for Cross Layer Design of Energy-Efficient Communication with QoS Provisioning in Multi-Hop Wireless Networks," Proc. 23rd Ann. Joint Conf. IEEE Computer and Comm. Soc., vol. 2, pp. 1446-56, Mar. 2004.

[9] X. Li and Z. Bao-yu, "Study on Cross-Layer Design and Power Conservation in Ad Hoc Network," Proc. Fourth Int'l Conf. Parallel and Distributed Computing, Applications and Technologies, pp. 324328, Aug. 2003.

[10] A. Safwat, H. Hassanein, and H. Mouftah, "Optimal Cross-Layer Designs for Energy-Efficient Wireless Ad Hoc and Sensor Networks," Proc. 22nd IEEE Int'l Performance Computing and Comm. Conf., Apr. 2003.

[11] "Wireless LAN Medium Access Control (MAC) and Physical Layer (PHY) Specifications," Technical Report Std 802.11, R2003, ANSI/IEEE, 1999.

[12] G. Bianchi, "Performance Analysis of IEEE 802.11 Distributed Coordination Function," IEEE J. Selected Areas in Comm., vol. 18, no. 3, pp. 535-547, Mar. 2000.

[13] I.S. Hwang and C.A. Chen, "Saturation Throughput Analysis in IEEE 802.11 DCF Using Semi-Markov Model," Int'l Math. J., vol. 1, nos. 5-8, pp. 289-296, 2006.

[14] K. Duffy, D. Malone, and D.J. Leith, "Modeling the 802.11 Distributed Coordination Function in Non-Saturated Heterogeneous Conditions," IEEE/ACM Trans. Networking, vol. 15, no. 1, pp. 159-172, Feb. 2007.

[15] F. Alizadeh-Shabdiz and S. Subramaniam, "Analytical Models for Single-Hop and Multi-Hop Ad Hoc Networks," Mobile Networks and Applications, vol. 11, no. 1, pp. 75-90, Feb. 2006.
[16] Y. Barowski, S. Biaz, and P. Agrawal, "Towards the Performance Analysis of IEEE 802.11 in Multi-Hop Ad-Hoc Networks," Proc. IEEE Wireless Comm. and Networking Conf., vol. 1, pp. 100-106, Mar. 2005.

[17] A. Tsertou and D.I. Laurenson, "Revisiting the Hidden Terminal Problem in a CSMA/CA Wireless Network," IEEE Trans. Mobile Computing, vol. 7, no. 7, pp. 817-831, July 2008.

[18] P.C. $\mathrm{Ng}$ and S.C. Liew, "Throughput Analysis of IEEE 802.11 Multi-Hop Ad Hoc Networks," IEEE/ACM Trans. Networking, vol. 15, no. 2, pp. 309-322, Apr. 2007.

[19] J. Chen, K. Sivalingam, and P. Agrawal, "Performance Comparison of Battery Power Consumption in Wireless Multiple Access Protocols," Wireless Networks, vol. 5, no. 6, pp. 445-460, Nov. 1999.

[20] R. Bruno, M. Conti, and E. Gregori, "Optimization of Efficiency and Energy Consumption in P-Persistent CSMA-Based Wireless LANs," IEEE Trans. Mobile Computing, vol. 1, no. 1, pp. 10-31, Jan. 2002.

[21] W.K. Kuo, "Energy Efficiency Modeling for IEEE 802.11 DCF System without Retry Limits," Computer Comm., vol. 30, no. 4, pp. 856-862, Feb. 2007.

[22] L. Ning, "A Power-Consumption Analysis for 802.11 DCF," Proc. Int'l Conf. Wireless Comm., Networking and Mobile Computing (WiCOM 06), pp. 1-4, Sept. 2006.

[23] M.M. Carvalho, C.B. Margi, K. Obraczka, and J.J. Garcia-LunaAceves, "Modeling Energy Consumption in Single-Hop IEEE 802.11 Ad Hoc Networks," Proc. 13th Int'l Conf. Computer Comm. and Networks, pp. 367-372, 2004.

[24] S. Ray and D. Starobinski, "On False Blocking in RTS/CTS-Based Multi-Hop Wireless Networks," IEEE Trans. Vehicular Technology, vol. 56, no. 2, pp. 849-862, Mar. 2007.

[25] B.R. Haverkort, Performance of Computer Communication Systems: A Model-Based Approach. Chichester, John Wiley \& Sons Ltd., 1998.

[26] C. Aydogdu, "An Analytical Model of IEEE 802.11 DCF for MultiHop Wireless Networks and Its Application to Goodput and Energy Analysis," PhD thesis, Bilkent Univ., Nov. 2010.

[27] D. Gross and C.M. Harris, Fundamentals of Queueing Theory, Wiley Series in Probability and Mathematical Statistics, second ed. John Wiley \& Sons Ltd., 1985.

[28] “The Network Simulator - ns-2," http://www.isi.edu/nsnam/ns, 2011.

[29] AT\&T, WaveMODEM 2.4GHz Data Manual, Release 2, 1995.

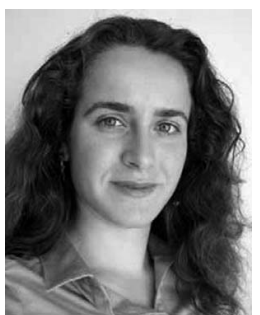

Canan Aydogdu received two BS degrees both with honors from the Electrical and Electronics Engineering Department and the Physics Department of Boğaziçi University, Istanbul, Turkey in 2001. She received the MS degree in 2003 and the PhD degree in 2010 in electrical and electronics engineering from Bilkent University, Ankara, Turkey. Her current work focuses on Bluetooth, IEEE 802.11, and energy-conserving protocols in wireless ad hoc and sensor networks. She is a student member of the IEEE.

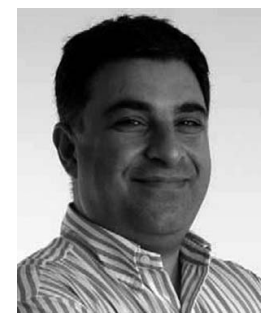

Ezhan Karasan received the BS degree from the Middle East Technical University, Ankara, Turkey, the MS degree from Bilkent University, Ankara, Turkey, and the $\mathrm{PhD}$ degree from Rutgers University, Piscataway, New Jersey, all in electrical engineering, in 1987, 1990, and 1995, respectively. During 1995-1996, he was a postdoctorate researcher at Bell Labs, Holmdel, New Jersey. From 1996 to 1998, he was a senior technical staff member at AT\&T Labs-Research Red Bank, New Jersey. He has been with the Department of Electrical and Electronics Engineering at Bilkent University since 1998. He is a member of the editorial board of Optical Switching and Networking Journal. He was the recipient of the 2004 Young Scientist Award from the Turkish Scientific and Technical Research Council (TUBITAK), the 2005 Young Scientist Award from Mustafa Parlar Foundation, and the Career Grant from TUBITAK in 2004. He is a member of the IEEE.

For more information on this or any other computing topic, please visit our Digital Library at www.computer.org/publications/dlib. 